\title{
DRTS Parsing with Structure-Aware Encoding and Decoding
}

\author{
Qiankun Fu ${ }^{1,2,3}$, Yue Zhang ${ }^{2,3}$, Jiangming Liu ${ }^{4}$ and Meishan Zhang ${ }^{5}$ \\ 1. Zhejiang University \\ 2. School of Engineering, Westlake University \\ 3. Institute of Advanced Technology, Westlake Institute for Advanced Study \\ 4. ILCC, University of Edinburgh \\ 5. School of New Media and Communication, Tianjin University, China \\ fqiankun@gmail.com and zhangyue@westlake.edu.cn \\ jiangming.liuded.ac.uk and mason.zms@gmail.com
}

\begin{abstract}
Discourse representation tree structure (DRTS) parsing is a novel semantic parsing task which has been concerned most recently. State-of-the-art performance can be achieved by a neural sequence-to-sequence model, treating the tree construction as an incremental sequence generation problem. Structural information such as input syntax and the intermediate skeleton of the partial output has been ignored in the model, which could be potentially useful for the DRTS parsing. In this work, we propose a structural-aware model at both the encoder and decoder phase to integrate the structural information, where graph attention network (GAT) is exploited for effectively modeling. Experimental results on a benchmark dataset show that our proposed model is effective and can obtain the best performance in the literature.
\end{abstract}

\section{Introduction}

Discourse representation tree structure (DRTS) is a form of discourse structure based on Discourse Representation Theory of Kamp and Reyle (1993), a popular theory of meaning representation (Kamp, 1981; Asher, 1993; Asher and Lascarides, 2003). It is designed to account for a variety of linguistic phenomena, including the interpretation of pronouns and temporal expressions within and across sentences. Correspondingly, as one type of discourse parsing, DRTS parsing (Liu et al., 2018) can be helpful for paragraph or document-level text understanding by converting DRS to tree-style DRTS. (Liu et al., 2019).

Figure 1 shows an example of DRTS, where the leaf nodes are discourse representation units (DRUs), upon which a discourse tree structure built. In particular, a DRU consists of several individual tuples, where each tuple denotes a relation inside the DRU. For example, there is a relationship
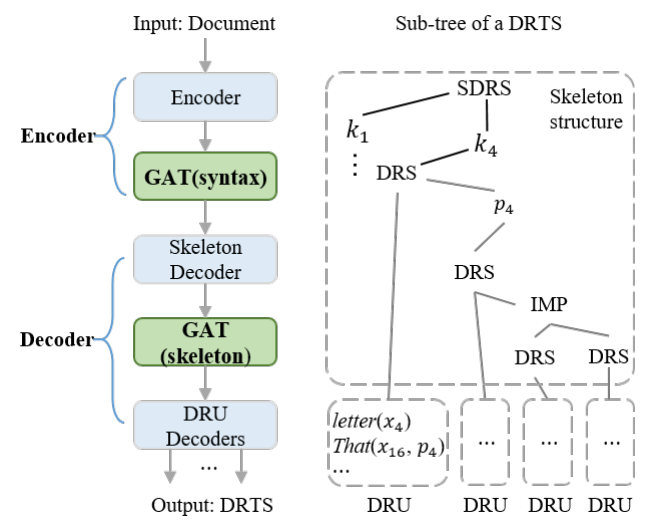

Figure 1: Left: Our proposed model with two structureaware module. Right: The DRTS for a clause in a document: "The letter $x_{4}$ warns Jewish women $x_{16}$ that they will suffer if they date Arab men. ${ }_{p_{4}}$ "'

"That" between the specific entity $x_{16}$ and a proposition $p_{4}$. The relationships between the DRUs are organized by a tree skeleton, which includes three types of nodes: the S(DRS) nodes to introduce DRU, the relation nodes for inter-DRU relationship, and the variable nodes, which are used to define $\mathrm{S}(\mathrm{DRS})$ (e.g., $p_{4}, k_{1}$ and $k_{4}$ ).

There have been only a few existing studies related to DRTS parsing (van Noord et al., 2018a,b). In particular, the end-to-end encoderdecoder model of Liu et al. (2019) gives the stateof-the-art performance, which converts the task into a sequence-to-sequence problem. The input sequence consists of words in paragraphs, encoded by a BiLSTM structure, and the output sequence is top-to-bottom depth-first traversal of the output DRTS tree, which is decoded incrementally with an attention-based LSTM feature representation module. During decoding, Liu et al. (2019) separate the skeleton generation and the DRU producing, as illustrated by Figure 1.

Although highly effective, the above model ig- 
nores some useful structure information in both the encoder and the decoder, which can be potentially useful for our task. Specifically, for encoding, syntax-based tree structure information has been demonstrated effective for a number of NLP tasks (Kasai et al., 2019; Li et al., 2018), including several other types of discourse parsing (Yu et al., 2018; $\mathrm{Li}$ et al., 2015). For decoding, the skeleton structure of DRTS can be also beneficial for our task. As a two-phase decoding strategy is exploited, the skeleton tree from the first phase could be helpful for DRU parsing of the second phase.

We propose to improve DRTS parsing by making use of the above structure information, modeling dependency-based syntax of the input sentences as well as the skeleton structure to enhance the baseline model of Liu et al. (2019) using Graph Attention Network (GAT) (Veličković et al., 2018), which has been demonstrated effective for tree/graph encoding (Huang and Carley, 2019; Linmei et al., 2019). In particular, we first derive dependency tree structures for each sentence in a paragraph from the Stanford Parser, and then encode them directly via one GAT module, which are fed as inputs for decoding. Second, after the first-state skeleton parsing is finished, we encode the skeleton structures by another GAT module, feeding the outputs for DRU parsing.

Following Liu et al. (2019), we conduct experiments on the Groningen Meaning Bank (GMB) dataset. Results show that structural information is highly useful for our task, bring a significantly better performance over the baseline. In particular, dependency syntax gives an improvement of $2.84 \%$ based on the standard evaluation metrics and the skeleton structure information gives a further improvement of $1.41 \%$. Finally, our model achieves $71.65 \% \mathrm{~F} 1$-score for the task, $4.25 \%$ better than the baseline model. Additionally, our model is also effective for sentence-level DRTS parsing, leading to an increase of $1.72 \%$ by the F1score by our final model. We release our code and best models at http://github.com/seanblank/ DRTSparsing for facilitating future research.

\section{Discourse Representation Tree (DRT)}

Formally, a DRT structure consists of two components according to the function: (1) the leaf nodes and (2) the tree skeleton (non-terminal nodes), respectively. Similar to other types of discourse representation methods, we have minimum semantic

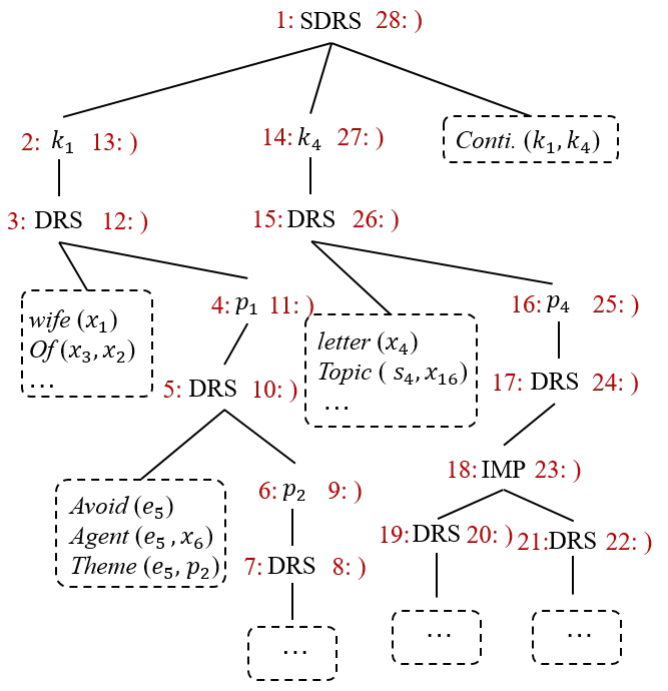

Figure 2: A full DRTS tree for document: " $k_{1}$ : At least 27 wives of Israeli rabbis have signed a letter urging Jewish women to avoid dating Arab men. $k_{4}$ : The letter warns Jewish women that they will suffer if they date Arab men." Red numbers indicate top-down depth-first order traversal of the DRTS skeleton.

units named by DRU, and then a discourse tree is built by the discourse relationships between these minimum units. Figure 2 shows the full tree version of Figure 1 in the introduction.

DRU. DRU serves as terminal nodes of a DRT structure, which is constituted by a set of unordered relation tuples, as shown by the below dashed components of the tree in Figure 1. A relation tuple consists of a relation $r$ and several arguments $v_{1} \cdots v_{n}$ in $r$, it can be denoted as $r\left(v_{1} \cdots v_{n}\right)$. Variables refer to entities $x$, events $e$, states $s$, time $t$, propositions $p$, segment $k$ and constants $c$. The relation is used to indicate the discourse connections among the inside variables. A total of 262 relation labels are defined in DRTS. One DRU may include unlimited relation tuples, which are all extracted from the corresponding text pieces.

Skeleton. The skeleton reflects the structural connection between DRUs. Nodes in a skeleton can be divided into three categories, including the (S)DRS nodes, the relation nodes and the variable nodes. In particular, (S)DRS nodes denotes a full semantically-completed node of discourse analysis. The relation node defines a specific discourse relationship over its covered (S)DRS nodes. DRTS has defined six types of DRS relations, including IMP (implication), OR (disjunction), DUP (duplex), POS (possibility), NEC (necessity) and 
NOT (negation), respectively, which is orthogonal to the relations inside the DRUs. The variable node assigns one (S)DRS node with a specific symbol. There are two types of variable nodes, namely proposition and segment. For example, in Figure 2, the root is a SDRS node, IMP is a relation nodes and $k_{1}, p_{4}$ denote the variable nodes.

\section{Baseline}

We take the multi-step encoder-decoder method of Liu et al. (2019) as the baseline model for DRTS parsing. First, an encoder is used to convert one input paragraph into neural vectors by using word embeddings as well as BiLSTMs, and then a multistep decoder is exploited to generate a full tree structure in a sequential manner incrementally.

\subsection{Encoder}

Given a paragraph, we concatenate all the sentences into one sequence, where each sentence is augmented with a start symbol $\langle s\rangle$ and an end token $\langle e\rangle$ at the front and end positions, respectively, obtaining a final input sequence for the paragraph $D=$ $\langle s\rangle, w_{1,1}, \ldots, w_{1, n_{1}},\langle e\rangle,\langle s\rangle, w_{2,1}, \ldots, w_{m, n_{m}},\langle e\rangle$. For simplicity, we use $D=w_{1}, \ldots, w_{n}$ to denote the sequence for short.

We use three different embedding representations to denote each word $w_{i}$ :

$$
\boldsymbol{v}_{i}=\boldsymbol{e}_{\text {rand }}\left(w_{i}\right) \oplus \boldsymbol{e}_{\text {pret }}\left(w_{i}\right) \oplus \boldsymbol{e}_{\mathrm{lem}}\left(w_{i}\right),
$$

where $\boldsymbol{e}_{\text {rand }}(\cdot)$, and $\boldsymbol{e}_{\text {pret }}(\cdot)$ denotes random and pretrained embeddings for current word, $e_{\mathrm{lem}}(\cdot)$ denotes the random embedding for current word lemma, and $\oplus$ denotes concatenation,

We then apply MLP over the word representations, and further use BiLSTM to encode the vector sequence:

$$
\begin{aligned}
\boldsymbol{x}_{1} \cdots \boldsymbol{x}_{n}=\operatorname{MLP}\left(\boldsymbol{v}_{1} \cdots \boldsymbol{v}_{n}\right) \\
\boldsymbol{H}^{\mathrm{enc}}=\boldsymbol{h}_{1} \cdots \boldsymbol{h}_{n}=\operatorname{BiLSTM}\left(\boldsymbol{x}_{1} \cdots \boldsymbol{x}_{n}\right),
\end{aligned}
$$

where $\boldsymbol{H}^{\text {enc }}=\boldsymbol{h}_{1} \cdots \boldsymbol{h}_{n}$ is the encoder output.

\subsection{Decoder}

We transform the DRTS structure into a sequence of symbols, so that the original DRTS can be restored from the symbol sequence as well. By this transformation, we can apply the sequence-to-sequence architecture for decoding. In particular, a two-stage strategy for the decoding is adapted, first generating the skeleton structure, and then generating the DRUs. The key step is the transformation strategies of the two stages.
Generating the skeleton structure. We define two types of symbols for each skeleton, where the first is the node label conjoined by a left bracket, indicting the start of traversal of the current node, and the second symbol is a right bracket, indicting the end of traversal of the current node. We exploit a top-down depth-first order to traverse the skeleton subtree, finishing a node traversal when all its child nodes have been finished. Figure 2 showed an example to illustrate the transformation. In this way, we can obtain a symbol sequence $Y^{\text {skt }}=$ $y_{1}^{\text {skt }}, \ldots, y_{s}^{\text {skt }}$ which is equivalent to the skeleton tree.

Generating the DRUs. After the skeleton is ready, we start the DRU generation process. The DRU nodes are only related to the (S)DRS nodes in the skeleton. Thus we generate DRU nodes one by one according to the (S)DRS nodes in the skeleton structure. For each DRU, we have two types of symbols, one for the relations and the other for the variables. We first generate all the relations and then generate the variables of each relation incrementally. ${ }^{1}$ In this way, we can obtain a sequence of $Y^{\mathrm{dru}}=y_{1}^{\mathrm{dru}}, \ldots, y_{t}^{\mathrm{dru}}$ for DRU generation. ${ }^{2}$

Sequence decoding. We follow the standard sequence-to-sequence architecture (Liu et al., 2018) to obtain the final sequence $Y=Y^{\text {skt }} Y^{\text {dru }}=$ $y_{1}^{\text {skt }}, \ldots, y_{s}^{\text {skt }} y_{1}^{\mathrm{dru}}, \ldots, y_{t}^{\mathrm{dru}}$ incrementally. At each step, we score the candidate next-step symbols based on current observations:

$$
\begin{aligned}
\boldsymbol{o}_{j}^{\mathrm{skt}} & =g^{\mathrm{skt}}\left(\boldsymbol{H}_{y_{<j}^{\text {skt }}}, \boldsymbol{H}^{\mathrm{enc}}\right), \\
\boldsymbol{o}_{k}^{\mathrm{dru}} & =g^{\mathrm{dru}}\left(\boldsymbol{H}_{y_{<k} s k}, \boldsymbol{H}^{\mathrm{skt}}, \boldsymbol{H}^{\mathrm{enc}}\right),
\end{aligned}
$$

where $\boldsymbol{H}^{\text {enc }}$ refers to the encoder outputs, $\boldsymbol{H}^{\text {skt }}$ and $\boldsymbol{H}^{\mathrm{dru}}$ denotes the outputs of skeleton decoder and the DRU decoder uses left-to-right LSTMs over $Y^{\text {skt }}$ and $Y^{\text {dru }}$, respectively, and $g^{\text {skt }}(\cdot)$ and $g^{\mathrm{dru}}(\cdot)$ are neural feature extraction functions for predicting skeleton and DRU symbols, respectively. Here we neglect the detailed description for $g^{\text {skt }}(\cdot)$ and $g^{\mathrm{dru}}(\cdot)$, which can be found in Liu et al. (2019).

Training. Given a set of labeled data, the model is trained to minimize average cross-entropy losses

\footnotetext{
${ }^{1}$ We follow a predefined order for relations. In fact, the order impacts little on the final influence.

${ }^{2}$ Our description is equivalent to Liu et al. (2019), who split this process into two steps (i.e., relation prediction and variable prediction). We merge the relation and variable predictions for brief.
} 

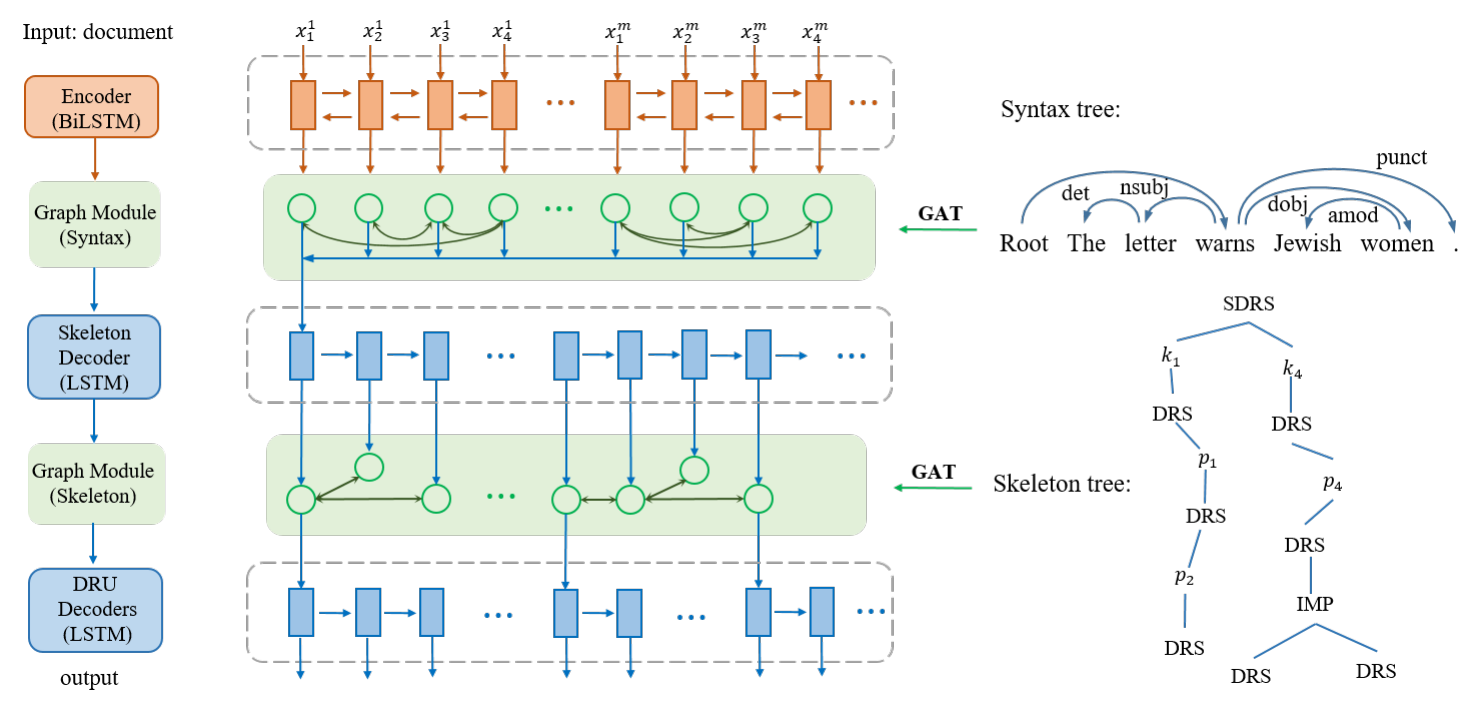

Figure 3: Model structure with two graph modules over RNN outputs. The hidden state for each word of the encoder is taken as the input node vector of the GAT module using syntax structure, and the output is fed into the skeleton decoder. The output of skeleton decoder is fed into the GAT module with the skeleton structure, and the output of GAT module is used to guide each DRU sequence generation.

over all individual symbol predictions:

$$
L(\theta)=-\frac{1}{N} \sum_{i} \log p_{y_{i}^{*}}
$$

where $\theta$ are the set of model parameters, $p_{y_{i}^{*}}$ denotes the output probability of $y_{i}^{*}$, which is computed by softmax over $\boldsymbol{o}_{i}, N$ is the total length of the output sequence.

\section{Structure-Aware Seq2Seq}

To represent the structure features, we use a GAT module on top of encoder and skeleton decoder stage to enhance the baseline model. The graph module is designed to learn non-local and nonsequential information from structural inputs. In this section, we first describe the GAT in detail and then illustrate its application on our task.

\subsection{Graph Attention Network}

Given a graph $G=(V, E)$, where each node $v_{i}$ has a initial vectorial representation, the GNN module enriches node representation with neighbor informations derived from the graph structure:

$$
\boldsymbol{H}^{l+1}=\operatorname{GNN}\left(\boldsymbol{H}^{l}, \boldsymbol{A} ; \boldsymbol{W}^{l}\right),
$$

where $\boldsymbol{H}^{l} \in \mathbb{R}^{n \times d}$ is the stacked hidden outputs for all nodes at layer $l\left(\boldsymbol{H}^{0}\right.$ denotes the input initial representations), $\boldsymbol{A} \in \mathbb{R}^{n \times n}$ denotes the graph adjacent matrix representation, and $\boldsymbol{W}^{l}$ is the parameter set of the GNN at layer $l$.
Different information aggregation functions lead to different GNN architectures. In particular, GAT uses the attention mechanism (Bahdanau et al., 2014) on graph neighbors, which has been demonstrated more effective than graph convolution neural network (GCN). The aggregation weights in GAT are computed by multi-head attention mechanism (Vaswani et al., 2017).

Specifically, given a node $i$ with a hidden representation $\boldsymbol{h}_{i}^{l}$ at layer $l$ and the its neighbors $\mathcal{N}_{i}$ as well as their hidden representations, a GAT updates the node's hidden representation at layer $l+1$ using multi-head attention:

$$
\boldsymbol{h}_{i}^{l+1}=\|_{k=1}^{K} \sigma\left(\sum_{j \in \mathcal{N}_{i}} \alpha_{i j}^{k} \boldsymbol{W}^{k} \boldsymbol{h}_{j}^{l}\right)
$$

where $\|$ represents concatenation, $\sigma$ is a sigmoid function, and $\boldsymbol{W}^{k}$ is the corresponding weight matrix of input linear transformation. $\alpha_{i j}^{k}$ are normalized attention coefficients computed by the $k$-th attention mechanism:

$$
\begin{aligned}
\alpha_{i j}^{k} & =\operatorname{SoftMAX}_{j}\left(e_{i j}\right) \\
& =\frac{\exp \left(e_{i j}\right)}{\sum_{k \in \mathcal{N}_{i}} \exp \left(e_{i k}\right)}
\end{aligned}
$$

where $e_{i j}$ is attention coefficient that indicate the importance of node $j$ to node $i$ computed by:

$$
e_{i j}=\operatorname{LeakyReLU}\left(f\left[\boldsymbol{W} \boldsymbol{h}_{i} \| \boldsymbol{W} \boldsymbol{h}_{j}\right]\right)
$$

$f(\cdot)$ is a single-layer feed-forward neural network, parameterized by a shared weight, $\boldsymbol{W}$ denotes a 


\begin{tabular}{lcccc}
\hline Section & \#Doc & \#Sent & AVG $_{\text {sent }}$ & AVG $_{\text {word }}$ \\
\hline Train & 7843 & 48599 & 6.2 & 135.3 \\
Devel & 991 & 6111 & 6.2 & 134.0 \\
Test & 1035 & 6469 & 6.3 & 137.2 \\
\hline
\end{tabular}

Table 1: Statistics on GMB document level benchmarks, $\mathbf{A V G}$ sent and $\mathbf{A V G}$ word denote the average number of sentences and words per document, respectively.

shared linear transformation and LeakyReLU is a non-linearity activation function.

\subsection{GAT for the Encoder}

On the encoder side, we equip the inputs with dependency syntax structures, which have been demonstrated helpful for closely-related tasks such as RST discourse parsing. A GAT module is used to represent the encoder output as mentioned in Section 4.1. We transform the document into a dependency graph represented by a undirected adjacent matrix using an off-the shelf dependency parser (Chen and Manning, 2014). The hidden states of each node is updated with a multi-layer GAT network on the adjacent matrix $\boldsymbol{A}$ :

$$
\boldsymbol{H}^{\mathrm{g} \text {-enc }}=\mathrm{GAT}^{\mathrm{enc}}\left(\boldsymbol{H}^{\mathrm{enc}} \oplus \boldsymbol{E}^{\mathrm{syn}}, \boldsymbol{A} ; \boldsymbol{W}\right),
$$

where $\boldsymbol{E}^{\text {syn }}$ is the embedding outputs of the syntactic labels in the dependency tree.

The learned representation $\boldsymbol{H}^{\mathrm{g} \text {-enc }}$ is used to substitute the original $\boldsymbol{H}^{\text {enc }}$ for predictions.

\subsection{GAT for the Decoder}

We further enhance the baseline model by exploiting the partial output after skeleton prediction step is finished. On one hand, the skeleton structures can guide for DRU parsing. On the other hand, the joint skeleton and DRU parsing can further help to rerank the skeleton predictions as well, since global skeleton representations are exploited.

Specifically, after all the skeleton nodes are generated, we construct a graph based on the nodes except the right parenthesis as shown in Figure 3. We use a GAT network on top of the hidden states to capture global structure information:

$$
\boldsymbol{H}^{\mathrm{g}-\mathrm{skt}}=\mathrm{GAT}^{\mathrm{skt}}\left(\boldsymbol{H}^{\mathrm{skt}} \oplus \boldsymbol{E}^{\mathrm{skt}}, \boldsymbol{A} ; \boldsymbol{W}\right),
$$

where $\boldsymbol{E}^{\text {skt }}$ is the embedding outputs of the node labels in the generated skeleton tree, and the global skeleton-aware representation $\boldsymbol{H}^{\text {g-skt }}$ is used instead of the original $\boldsymbol{H}^{\text {skt }}$ for future predictions.

\section{Experiments}

\subsection{Data and Settings}

Data We conduct experiments on the benchmark GMB dataset, which provides a large collection of English texts annotated with Discourse Representation Structures (Bos et al., 2017). We follow Liu et al. (2019) using the processed tree-based DRTS format, and focus on document-level parsing. The data statistics are shown in Table 1.

Hyperparameters We exploit the same hyperparameters as Liu et al. (2019) for fair comparison. In particular, we use the same pre-trained 100dimensional word embeddings, which are trained on the AFP portion of the English Gigaword corpus. The sizes of random word and lemma embeddings are set to 300 and 100, respectively. The hidden sizes of BiLSTM modules in encoder and decoder are set to 300 and 600, respectively. In addition, the BiLSTM layer sizes of encoder and decoder are respectively 2 and 1 . The hidden size of GAT modules is set to 300 and 600 for encoder and decoder, respectively.

\subsection{Evaluation}

Following Liu et al. (2019), we adopt the Counter (van Noord et al., 2018a) tool to evaluate our final experimental results. In particular, we first transform the DRTS into a clause format and then run the standard evaluation script to obtain the F1-scores of our results compared with the gold-standard clause form. Note that COUNTER is computationally expensive, requiring more than 50 hours for the entire test dataset by using more than 100 threads. To facilitate development and analysis experiments, we suggest three alternatives for evaluation particularly for development experiments:

(1) BLEU: a standard BLEU (Papineni et al., 2002) value is adopted as the metric to evaluate the resulting node sequence against the gold-standard output, since we model the task as a sequence-to-sequence task.

(2) Skeleton: The bracket scoring method of constituent parsing is exploited to evaluate the skeleton performance, by regarding terminal DRU nodes as words in comparison with a constituent tree. ${ }^{3}$

\footnotetext{
${ }^{3}$ https://nlp.cs.nyu.edu/evalb/
} 


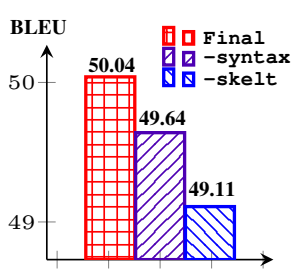

(a) structure labels

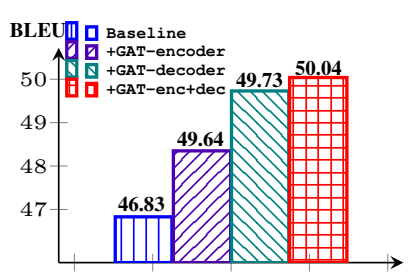

(b) GAT modules
Figure 4: Feature ablation experiments.

(3) Tuple: The F1-score of tuple-level matching is exploited to measure the DRU performance, since the basic units inside a DRU are tuples of relation-variable functions. Exact matching is adopted considering variable orders.

The BLEU is used for development and the Skeleton and Tuple are used for analysis.

\subsection{Development Experiments}

We conduct experiments on the development dataset to understand the key factors of our proposed model.

Impact of structure labels Syntactic arcs and skeleton labels are embedded and concatenated to the embedding of the current node when using GAT to model the tree structure. We conduct a comparison to examine their effectiveness in our model. Figure 4(a) shows the results. We can see that a performance degradation occurs without these label embeddings. In particular, BLEU score drops by 0.4 without syntax label embeddings and 0.93 without skeleton label embeddings, which shows that modeling label information improves unfixed skeleton tree structure even more.

Impact of GAT setting As our proposed modules involve a $l$-layer GAT, we investigate the effect of the layer number $l$ on the dev set as shown in Table 2. In particular, we vary the value of $l$ in the set $\{1,2,3,4,5\}$ and measure the corresponding BLEU scores. The structural-aware model equipped with GAT achieves the best performance when $l$ is 2 , which justifies the selection on the number of layers in the experimental setting section. Moreover, a dropping trend on both metrics is present as $l$ increases. For a larger $l$, the GAT module becomes more difficult to train due to larger amounts of parameters. One intuitive reason is that each layer of the GAT module aggregates

\begin{tabular}{lcccc}
\hline Model & BLEU & & Model & BLEU \\
\cline { 1 - 2 } Head $=1$ & 48.76 & & layers $=1$ & 49.11 \\
Head $=2$ & 49.48 & & layers $=2$ & $\mathbf{5 0 . 0 4}$ \\
Head $=3$ & 50.01 & & layers $=3$ & 49.72 \\
Head $=4$ & $\mathbf{5 0 . 0 4}$ & & layers $=4$ & 49.01 \\
Head $=5$ & $\mathbf{5 0 . 0 4}$ & & layers $=5$ & 48.54 \\
\hline
\end{tabular}

Table 2: GAT settings results on development set.

\begin{tabular}{c|cc}
\hline Model & BLEU & exact F1 \\
\hline $\begin{array}{c}\text { Liu et al. (2019) } \\
\text { (baseline) }\end{array}$ & 46.86 & 66.56 \\
\hline GAT-encoder & 48.24 & 69.40 \\
GAT-decoder & 50.04 & 70.81 \\
GAT-enc+dec & $\mathbf{5 0 . 1 6}$ & $\mathbf{7 1 . 6 5}$ \\
\hline \hline Tree-LSTM & 48.36 & 69.66 \\
GCN & 49.88 & 70.72 \\
\hline
\end{tabular}

Table 3: Final results on the test dataset.

the direct neighbor information of a node. After 2 layers, each node can obtain sufficient information, and further more layers can bring noise.

We make comparison with multi-head attention, varying the heads in the set $\{1,2,3,4,5\}$ and checking the corresponding BLEU scores. Theoretically, the larger the number of heads, the better the performance of the model. As can be seen in Table 2 , when the number of heads exceeds 4 , the performance becomes relatively stable. We thus choose the head to be 4 for the remaining experiments.

Influence of the encoder and decoder GAT modules As shown in Figure 4(b), without using structure information, the baseline encoder-decoder (Liu et al., 2019) model gives a development BLEU of 46.83. Adding a GAT module to the encoder as described in Section 4.2 increases the BLEU score to 48.35 , demonstrating the usefulness of syntax-aware module. Furthermore, adding a GAT module to the decoder as described in Section 4.3 improves the performance to 49.73 , which shows that our skeleton structure model is useful. Finally, a combination of both gives a 50.04 BLEU score.

\subsection{Final Results}

Table 3 shows the final results on the GMB test dataset. We report performances of the baseline and various tree-structure systems using the exact F1-score by COUNTER in addition to BLEU. The observations are consistent with the development set. Our final model, the joint GAT-enc+dec model, 


\begin{tabular}{c|cc}
\hline Model & BLEU & exact F1 \\
\hline $\begin{array}{c}\text { Liu et al. (2019) } \\
\text { (baseline) }\end{array}$ & 64.96 & 77.85 \\
\hline GAT-encoder & 66.02 & 78.22 \\
GAT-decoder & 66.69 & 79.14 \\
GAT-enc+dec & 68.14 & 79.94 \\
\hline Liu et al. (2018) & 57.61 & 68.72 \\
\hline
\end{tabular}

Table 4: Results on the sentence-level dataset.

achieves competitive performance, with a exact F1score of $71.65 \%$. Our GAT enhanced models outperform the state-of-the-art model. For the vanilla encoder-decoder model, our GAT-encoder obtains a absolute improvement of $2.84 \%$ exact $\mathrm{F} 1$-score, which demonstrates that modeling syntax information is useful. The GAT decoder improves the performance to $70.81 \%$, giving a $4.25 \%$ promotion, which indicates that the skeleton structure is helpful to DRTS parsing.

As shown in Table 3, Tree-LSTM and GCN based systems also give competitive results to the state-of-the-art baseline model, which again demonstrates the effectiveness of modeling tree structures. GCN achieves better performance than Tree-LSTM by $1.06 \%$, which can be because the GNN-based model obtains global information during layer stacking, but Tree-LSTM can only capture local structural information. GAT performs better than GCN by $0.84 \%$, showing that GAT is a competitive choice of GNN. Consistent with observations of BLEU scores, our proposed GAT-enc+dec model shows the best performance on both evaluation metrics.

In addition, we perform experiments on sentence-level datasets as shown in Table 4 as well, following Liu et al. (2019). We use the same setup as the document-level structure-aware model. As shown, both the GAT encoder and decoder can bring better results (i.e., $0.37 \%$ and $1.29 \%$ by the GAT encoder and decoder, respectively), and their combination can give further improvements (i.e., $0.80 \%$ over the GAT-decoder) significantly, which are consistent with the findings of the documentlevel parsing. Finally, the sentence-level performance reaches $79.94 \%$, a new state-of-the-art score. The results demonstrate that our model is also applicable to sentence-level DRTS parsing.

Interestingly, we find that the BLEU metric is highly indicative of model performance. Based on the observed pair of values on the test results, we

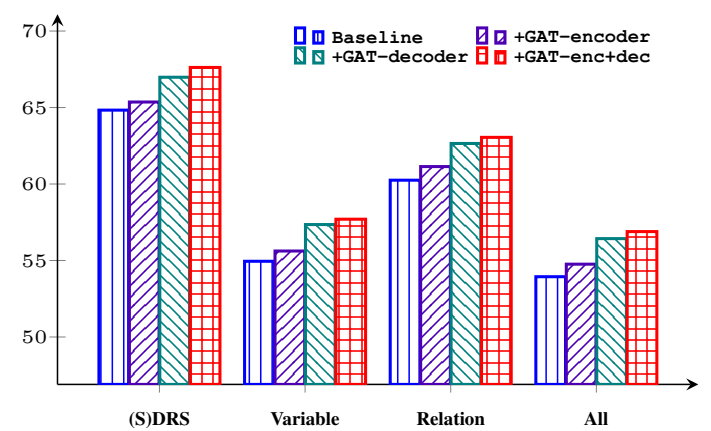

Figure 5: Skeleton-level evaluation F1 (\%) results.

\begin{tabular}{lcccc}
\hline Model & Rel $_{- \text {var }}$ & Rel & Unary & Binary \\
\hline baseline & 64.13 & 34.80 & 39.21 & 26.38 \\
GAT-encoder & 66.67 & 36.08 & 40.98 & 27.10 \\
GAT-decoder & 68.32 & 36.41 & 42.34 & 27.22 \\
GAT-enc+dec & $\mathbf{6 8 . 9 7}$ & $\mathbf{3 7 . 0 9}$ & $\mathbf{4 3 . 7 6}$ & $\mathbf{2 7 . 7 4}$ \\
\hline
\end{tabular}

Table 5: Relation-level evaluation F1 (\%) results.

are able to approach the correction between BLEU and COUNTER by a line appropriately, demonstrating a faithful alignment to the COUNTER metric. The observation indicates that the BLEU is also a good metric for the task. Noticeably, one advantage of the BLEU is that the metric calculation is much faster (i.e., only several seconds) than the exact-F1 score, since the latter one consumes at least 24 hours as well as $100 \mathrm{G}+$ memory for the evaluation of the test dataset.

\subsection{Analysis}

We conduct analysis to examine benefits by the structural-aware model. As the decoding process is decomposed into two steps, we examine the respective gains with respect to the two components, namely skeleton prediction and DRU parsing.

Influence on Skeleton Prediction The bracket scoring metric suggested in Section 5.2 is used to measure the performance of skeleton prediction. Figure 5 shows the F1-scores with respect to node types, which are categorized into three types (Section 2), namely (S)DRS, relation and variable. In addition, the overall performance is reported as well. First, we can see that the (S)DRS nodes can achieve the best performance across the three types, the relation nodes rank the second and the variable type has the worst performance. This indicates the relative difficulty in parsing the three types of nodes. In particular, locating a DRU is relatively simpler as (S)DRS connects with DRU directly, 

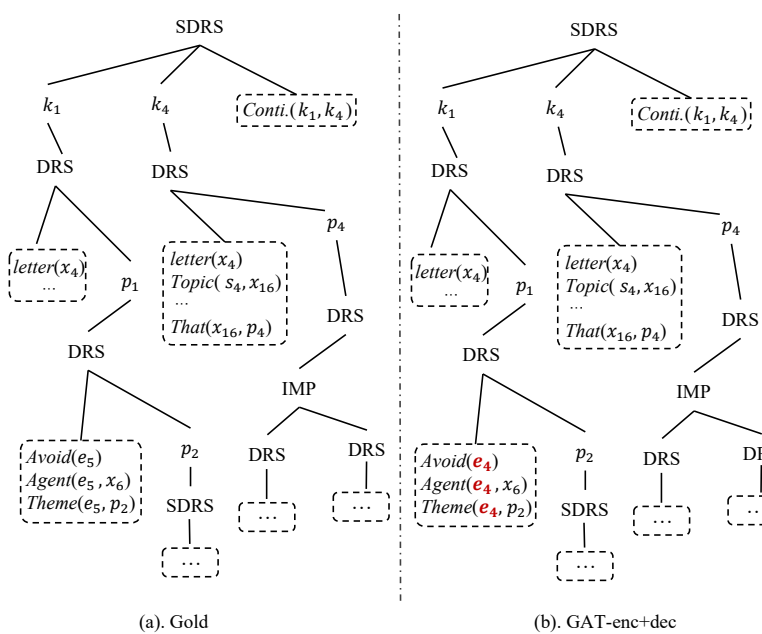

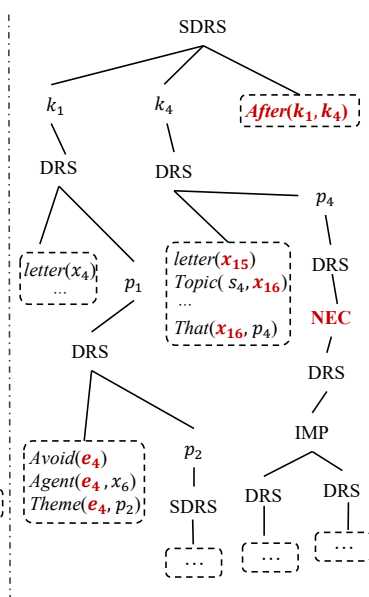

(c): GAT-decoder

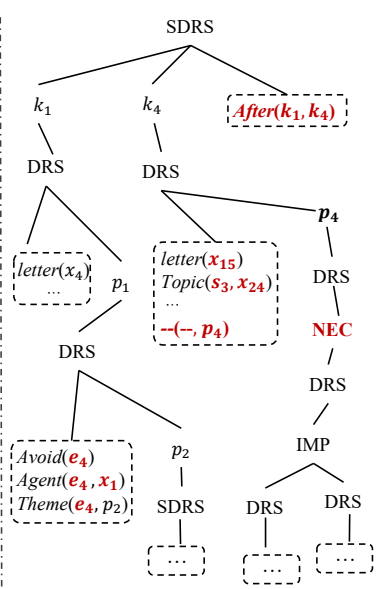

(d). Baseline model

Figure 6: Discourse representation tree structure examples generated by different models: " $k_{1}$ : At least 27 wives of Israeli rabbis have signed a letter urging Jewish women to avoid dating Arab men. $k_{4}$ : The letter warns Jewish women that they will suffer if they date Arab men."

followed by the coarse-grained discourse relations over the DRUs, while variable nodes are much more difficult since the order matters much (i.e., the subscript number in the variable). Second, the tendencies in terms of different models on the three categories are the same as the overall tendency, where our final model can bring the best skeleton performance, and the baseline shows the worst performance. The observation demonstrates the robustness of our proposed structural-aware model: we can achieve consistently better performances on all the types over the baseline.

Influence on relation tuples inside DRUs Further we analyze the model performance on DRU parsing. A strict matching strategy on the relation tuples inside DRUs is used to measure the performance, as described in Section 5.2. Table 5 shows the performances, where the F1-scores of the overall matching, only relation matching as well as unary and binary relation tuples are reported. ${ }^{4}$ First, we can find that the overall exact matching F1-score is rather low (below 40). When considering the relation performance ignoring the variables, the final F1-score reaches, with an increase of 31.88 , which indicates that variable recognition is extremely difficult. Variables in DURs are similar to the variable nodes in skeleton, however the scale of the inside DRU variables is much larger. We further categorize the relation tuples by their number of variables. The unary tuples (i.e. tuples consist of only one

\footnotetext{
${ }^{4}$ There are no relations containing more than two variables according to the corpus statistics.
}

variable node) can obtain better performance than the binary tuples (i.e. tuples consist of two variable nodes), which is reasonable. In addition, we look into the performance in terms of different models. We can see that all structural-aware models can obtain better performances than the baseline on all settings, demonstrating the effectiveness of our proposed models. In particular, the GAT-decoder demonstrates relatively higher performance compared to GAT-encoder, which is consistent with the results observed in Table 3 . As expected, the final joint GAT-enc+dec model obtain a better score than both of individual GAT models.

Case study Figure 6 shows one case study to illustrate the gains of our proposed models over the baseline model, where the detailed differences are highlighted with red color. As shown, the baseline model is already able to obtain a strong results with linguistically-motivated copy strategies, constraint-based inference and so on. However, without structural-aware information, the model is ineffective to handle several implicit long-distance dependencies.

For example, the relation of "That $\left(x_{16}, p_{4}\right)$ " is unable to be recognized by the baseline model, while the models with structural-aware GAT decoder can get it correctly. The major reason is that the structural-aware decoder can transmit the information from $p_{4}$ to its parent node, which can facilitate the next-step generation of the parent node.

On the other hand, the syntactic information from the input sentences can help the first-step 
skeleton disambiguation. For example, as shown in Figure 6, the models without GAT-encoder can misclassify the relations between $k_{1}$ and $k_{4}$, which is the discourse relation between the input two short sentences. The major reason of the misleading may be possibly due to the word "if" in the second sentence, which is one indicator for the After relation. When the syntactic information is encoded by the GAT encoder, the GAT-enc+dec model can learn the fined-grained dependency reduced by the word "if", and thus is able to obtain the accurate relation of the two sentences (i.e., Conti.)

\section{Related work}

Discourse parsing is one important topic in the NLP. There are several main types of discourse parsing tasks in the literature, including rhetorical structure theory (RST; MANN and Thompson, 1988) based parsing, centering theory (CT; Grosz et al., 1995; Barzilay and Lapata, 2008) based parsing and DRT based parsing in this study.

Discourse Representation Theory (DRT) based parsing is a relatively classic, yet not fully researched semantic analysis task because of its complexity. Le and Zuidema (2012) present the first work of a data-driven DRT parser, using a graphbased representation of DRT structures. Recently, van Noord et al. (2018b) apply the idea of neural machine translation for graph-based DRT parsing, achieving impressing performance. These studies only focus on sentence-level DRT representations, as the complexity would increase much at the paragraph level. In contrast, we investigate the paragraph level DRT parsing.

DRTS parsing simplifies graphs into trees. There are two existing papers in this line. Liu et al. (2018) are the first to work on DRTS parsing, who propose an end-to-end sequence-to-sequence model for the task. Further, Liu et al. (2019) improve the model by suggesting several effective strategies including supervised attention, copying from alignments, and constraint-based inference. In this work, we improve DRTS parsing instead of Liu et al. (2019) with two types of structure information.

Syntax information has been widely exploited for NLP tasks. Seminal work exploits discrete features designed by experts (Feng and Hirst, 2014; Heilman and Sagae, 2015). Recently, a range of neural modules have been proposed to encode syntax, such as Tree-LSTM (Tai et al., 2015; Zhu et al.; Teng and Zhang, 2016), Tree-CNN (Roy et al., 2020) and the recently proposed implicit approaches (Yin et al., 2018; Zhang et al., 2019). Syntax has been demonstrated effective for RST based discourse parsing as well (Yu et al., 2018). Our work is to build a syntax tree-aware model and we are the first to use syntax for DRT based discourse parsing.

GNN has received increasing interests for its strong capability of encoding structural information (Kipf and Welling, 2016; Bastings et al., 2017; Zhang et al., 2018; Zhang and Zhang, 2019; Song et al., 2018). GAT is one representative model, which demonstrates success in a number of NLP tasks (Huang and Carley, 2019; Linmei et al., 2019). In this work, we exploit GAT to represent treestructural information for DRTS parsing.

\section{Conclusion}

We investigated the representation of structural information for discourse representation tree structure parsing, showing that a graph neural network can bring significant improvements. In particular, we use GAT for representing syntax in encoding, and representing a structural backbone for decoding. Experiments on the standard GMB dataset show that our method is high effective, achieving the best results in the literature.

\section{Acknowledgments}

We thank all reviewers for the valuable comments, which greatly help to improve the paper. This work is supported by the National Natural Science Foundation of China (NSFC No. 61976180), the funds of Beijing Advanced Innovation Center for Language Resources (No. TYZ19005) and the Westlake University and Bright Dream Joint Institute for Intelligent Robotics. Meishan Zhang is the corresponding author.

\section{References}

Nicholas Asher. 1993. Reference to Abstract Objects in Discourse. Kluwer.

Nicholas Asher and Alex Lascarides. 2003. Logics of Conversation. Cambridge University Press.

Dzmitry Bahdanau, Kyunghyun Cho, and Yoshua Bengio. 2014. Neural machine translation by jointly learning to align and translate. CoRR, abs/1409.0473.

Regina Barzilay and Mirella Lapata. 2008. Modeling local coherence: An entity-based approach. Сотриtational Linguistics, 34(1):1-34. 
Joost Bastings, Ivan Titov, Wilker Aziz, Diego Marcheggiani, and Khalil Sima'an. 2017. Graph convolutional encoders for syntax-aware neural machine translation. In Proceedings of the 2017 Conference on Empirical Methods in Natural Language Processing, pages 1957-1967, Copenhagen, Denmark. Association for Computational Linguistics.

Johan Bos, Valerio Basile, Kilian Evang, Noortje J. Venhuizen, and Johannes Bjerva. 2017. The Groningen Meaning Bank, pages 463-496. Springer Netherlands, Dordrecht.

Danqi Chen and Christopher Manning. 2014. A fast and accurate dependency parser using neural networks. In Proceedings of the 2014 Conference on Empirical Methods in Natural Language Processing (EMNLP), pages 740-750, Doha, Qatar. Association for Computational Linguistics.

Vanessa Wei Feng and Graeme Hirst. 2014. A lineartime bottom-up discourse parser with constraints and post-editing. In Proceedings of the 52nd Annual Meeting of the Association for Computational Linguistics (Volume 1: Long Papers), pages 511521, Baltimore, Maryland. Association for Computational Linguistics.

Barbara J. Grosz, Aravind K. Joshi, and Scott Weinstein. 1995. Centering: A framework for modeling the local coherence of discourse. Computational Linguistics, 21(2):203-225.

Michael Heilman and Kenji Sagae. 2015. Fast rhetorical structure theory discourse parsing. ArXiv, abs/1505.02425.

Binxuan Huang and Kathleen Carley. 2019. Syntaxaware aspect level sentiment classification with graph attention networks. In Proceedings of the 2019 Conference on Empirical Methods in Natural Language Processing and the 9th International Joint Conference on Natural Language Processing (EMNLP-IJCNLP), pages 5468-5476, Hong Kong, China. Association for Computational Linguistics.

Hans Kamp. 1981. A theory of truth and semantic representation. In P. Portner and B. H. Partee, editors, Formal Semantics - the Essential Readings, pages 189-222. Blackwell.

Hans Kamp and Uwe Reyle. 1993. From Discourse to Logic. Dordrecht: Kluwer Academic Publishers.

Jungo Kasai, Dan Friedman, Robert Frank, Dragomir Radev, and Owen Rambow. 2019. Syntax-aware neural semantic role labeling with supertags. In Proceedings of the 2019 Conference of the North American Chapter of the Association for Computational Linguistics: Human Language Technologies, Volume 1 (Long and Short Papers), pages 701-709, Minneapolis, Minnesota. Association for Computational Linguistics.

Thomas N Kipf and Max Welling. 2016. Semisupervised classification with graph convolutional networks. arXiv preprint arXiv:1609.02907.
Phong Le and Willem Zuidema. 2012. Learning compositional semantics for open domain semantic parsing. In Proceedings of COLING 2012, pages 15351552, Mumbai, India. The COLING 2012 Organizing Committee.

Jiwei Li, Thang Luong, and Dan Jurafsky. 2015. A hierarchical neural autoencoder for paragraphs and documents. In Proceedings of the 53rd Annual Meeting of the Association for Computational Linguistics and the 7th International Joint Conference on Natural Language Processing (Volume 1: Long Papers), pages 1106-1115, Beijing, China. Association for Computational Linguistics.

Zuchao Li, Shexia He, Jiaxun Cai, Zhuosheng Zhang, Hai Zhao, Gongshen Liu, Linlin Li, and Luo Si. 2018. A unified syntax-aware framework for semantic role labeling. In Proceedings of the 2018 Conference on Empirical Methods in Natural Language Processing, pages 2401-2411, Brussels, Belgium. Association for Computational Linguistics.

Hu Linmei, Tianchi Yang, Chuan Shi, Houye Ji, and Xiaoli Li. 2019. Heterogeneous graph attention networks for semi-supervised short text classification. In Proceedings of the 2019 Conference on Empirical Methods in Natural Language Processing and the 9th International Joint Conference on Natural Language Processing (EMNLP-IJCNLP), pages 48204829 , Hong Kong, China. Association for Computational Linguistics.

Jiangming Liu, Shay B. Cohen, and Mirella Lapata. 2018. Discourse representation structure parsing. In Proceedings of the 56th Annual Meeting of the Association for Computational Linguistics (Volume 1: Long Papers), pages 429-439, Melbourne, Australia. Association for Computational Linguistics.

Jiangming Liu, Shay B. Cohen, and Mirella Lapata. 2019. Discourse representation parsing for sentences and documents. In Proceedings of the 57th Annual Meeting of the Association for Computational Linguistics, pages 6248-6262, Florence, Italy. Association for Computational Linguistics.

WILLIAM MANN and Sandra Thompson. 1988. Rethorical structure theory: Toward a functional theory of text organization. Text, 8:243-281.

Rik van Noord, Lasha Abzianidze, Hessel Haagsma, and Johan Bos. 2018a. Evaluating scoped meaning representations. In Proceedings of the Eleventh International Conference on Language Resources and Evaluation (LREC 2018), Miyazaki, Japan. European Language Resources Association (ELRA).

Rik van Noord, Lasha Abzianidze, Antonio Toral, and Johan Bos. 2018b. Exploring neural methods for parsing discourse representation structures. Transactions of the Association for Computational Linguistics, 6:619-633. 
Kishore Papineni, Salim Roukos, Todd Ward, and Wei Jing Zhu. 2002. Bleu: a method for automatic evaluation of machine translation.

Deboleena Roy, Priyadarshini Panda, and Kaushik Roy. 2020. Tree-cnn: A hierarchical deep convolutional neural network for incremental learning. Neural Networks, 121:148 - 160 .

Linfeng Song, Yue Zhang, Zhiguo Wang, and Daniel Gildea. 2018. A graph-to-sequence model for AMRto-text generation. In Proceedings of the 56th Annual Meeting of the Association for Computational Linguistics (Volume 1: Long Papers), pages 16161626, Melbourne, Australia. Association for Computational Linguistics.

Kai Sheng Tai, Richard Socher, and Christopher D. Manning. 2015. Improved semantic representations from tree-structured long short-term memory networks. In Proceedings of the 53rd Annual Meeting of the Association for Computational Linguistics and the 7th International Joint Conference on Natural Language Processing (Volume 1: Long Papers), pages 1556-1566, Beijing, China. Association for Computational Linguistics.

Zhiyang Teng and Yue Zhang. 2016. Bidirectional treestructured lstm with head lexicalization.

Ashish Vaswani, Noam Shazeer, Niki Parmar, Jakob Uszkoreit, Llion Jones, Aidan N Gomez, Ł ukasz Kaiser, and Illia Polosukhin. 2017. Attention is all you need. In I. Guyon, U. V. Luxburg, S. Bengio, H. Wallach, R. Fergus, S. Vishwanathan, and R. Garnett, editors, Advances in Neural Information Processing Systems 30, pages 5998-6008. Curran Associates, Inc.

Petar Veličković, Guillem Cucurull, Arantxa Casanova, Adriana Romero, Pietro Liò, and Yoshua Bengio. 2018. Graph attention networks. In International Conference on Learning Representations.

Pengcheng Yin, Chunting Zhou, Junxian He, and Graham Neubig. 2018. StructVAE: Tree-structured latent variable models for semi-supervised semantic parsing. In Proceedings of the 56th Annual Meeting of the Association for Computational Linguistics (Volume 1: Long Papers), pages 754-765, Melbourne, Australia. Association for Computational Linguistics.

Nan Yu, Meishan Zhang, and Guohong Fu. 2018. Transition-based neural RST parsing with implicit syntax features. In Proceedings of the 27th International Conference on Computational Linguistics, pages 559-570, Santa Fe, New Mexico, USA. Association for Computational Linguistics.

Liwen Zhang, Kewei Tu, and Yue Zhang. 2019. Latent variable sentiment grammar. In Proceedings of the 57th Annual Meeting of the Association for Computational Linguistics, pages 4642-4651, Florence, Italy. Association for Computational Linguistics.
Yuan Zhang and Yue Zhang. 2019. Tree communication models for sentiment analysis. In Proceedings of the 57th Annual Meeting of the Association for Computational Linguistics, pages 3518-3527, Florence, Italy. Association for Computational Linguistics.

Yue Zhang, Qi Liu, and Linfeng Song. 2018. Sentencestate LSTM for text representation. In Proceedings of the 56th Annual Meeting of the Association for Computational Linguistics (Volume 1: Long Papers), pages 317-327, Melbourne, Australia. Association for Computational Linguistics.

Xiaodan Zhu, Parinaz Sobhani, and Hongyu Guo. Long short-term memory over recursive structures. In Proceedings of International Conference on $\mathrm{Ma}$ chine Learning. 